\title{
A qualidade de sono de fisioterapeutas de um hospital público durante a pandemia de Covid-19
}

\author{
The quality of sleep of physiotherapists in a public \\ hospital during the Covid-19 pandemic
}

\author{
Jueline da Silva Santos ${ }^{1}$ (1) \\ Camila de Araújo Lima² ${ }^{2}$ \\ Whelen de Sousa Moreira ${ }^{3}$ (1) \\ Paulo Nixon Cardoso Monteiro 4 (1) \\ Mylena Cardoso Sales 5 \\ Jaíne de Sousa Lima ${ }^{6}$ \\ Carlos Eduardo Nunes Vieira ${ }^{7}$ \\ ${ }^{1-4}$ Hospital Universitário da Universidade Federal do Piauí (Teresina). Piauí, Brasil. juelinesilva@hotmail.com, kmilalima7@gmail.com, \\ whellen_2011@hotmail.com, paulonixon17@gmail.com \\ ${ }^{5-7}$ Centro Universitário Maurício de Nassau (Teresina).Piauí, Brasil. mylena.mylenasales@hotmail.com, limajaine10@gmail.com, cadu.nunes11@hotmail.com \\ ${ }^{8}$ Autora para correspondência. Hospital Universitário da Universidade Federal do Piauí (Teresina). Piauí, Brasil. luanagabrielle@yahoo.com.br
}

\begin{abstract}
RESUMO | INTRODUÇÃO: A pandemia de covid-19 tornou necessário estudos sobre o impacto da pandemia e das novas rotinas de trabalho impostas ao trabalhador no estado de saúde de profissionais de saúde, em especial os fisioterapeutas hospitalares. OBJETIVO: Avaliar a qualidade de sono e sonolência diurna de profissionais de fisioterapia hospitalar durante o período de pandemia do covid-19. MATERIAIS E MÉTODOS: Pesquisa observacional, transversal, prospectiva em um hospital público do nordeste brasileiro. A pesquisa teve como público-alvo fisioterapeutas hospitalares atuantes ou não em setores covid durante a pandemia de covid-19. Foram aplicados os instrumentos Índice de Qualidade do Sono de Pittsburgh (IQSP), Escala de Sonolência de Epworth (ESE) e questionários com características demográficas, trabalho, saúde e percepção de estresse. RESULTADOS: Participaram do estudo 45 fisioterapeutas e foi observado que $62,2 \%$ eram do sexo feminino, $66,7 \%$ relataram trabalhar $60 \mathrm{~h}$ por semana e $55,6 \%$ trabalharam em setor covid e não covid. Observou-se elevada frequência de má qualidade do sono $(68,9 \%)$ independente de carga horária ou setor de trabalho. Houve maior prevalência de sonolência diurna excessiva $(43,3 \%)$ entre os fisioterapeutas que trabalhavam $60 \mathrm{~h}$ por semana. CONCLUSÃO: Os fisioterapeutas hospitalares de uma instituição pública têm má qualidade do sono e aqueles que trabalham com maior carga horária apresentam maior prevalência de sonolência diurna excessiva.
\end{abstract}

PALAVRAS-CHAVE: Transtornos do Sono-Vigília. Estresse Ocupacional. Sonolência. Coronavírus.

\begin{abstract}
INTRODUCTION: The covid-19 pandemic has made it necessary to study the impact of the pandemic and the new work routines imposed on workers on the health status of health professionals, especially hospital-based physical therapists. OBJECTIVE: To evaluate the quality of sleep and daytime sleepiness of hospital-based physical therapists during the covid-19 pandemic. MATERIALS AND METHODS: This is an observational, cross-sectional, prospective study conducted in a public hospital in northeastern Brazil. The research had as target audience, hospital-based physical therapists working or not in covid sectors during the covid-19 pandemic. We applied the Pittsburgh Sleep Quality Index (PSQI), the Epworth Sleepiness Scale (ESS), and questionnaires with demographic, work, health, and stress perception characteristics. RESULTS: Forty-five physical therapists participated in the study, and it was observed that $62.2 \%$ were female, $66.7 \%$ reported working $60 \mathrm{~h}$ per week, and $55.6 \%$ worked in the covid and non-covid sectors. A high frequency of poor sleep quality (68.9\%) was observed regardless of workload or work sector. In addition, there was a higher prevalence of excessive daytime sleepiness (43.3\%) among physical therapists who worked 60h per week. CONCLUSION: Hospital-based physical therapists in a public institution have poor sleep quality, and those who work more hours have a higher prevalence of excessive daytime sleepiness.
\end{abstract}

KEYWORDS: Sleep Wake Disorders. Occupational Stress. Sleepiness. Coronavirus. 


\section{Introdução}

As exigências da sociedade moderna fazem com que a população não durma o suficiente diariamente. Enfatizando os trabalhadores da área da saúde, estes exercem suas atividades profissionais em formato de plantões, em turnos noturnos e rotativos, além dos vários vínculos empregatícios assumidos para gerar renda financeira compatível com suas necessidades pessoais. Toda essa rotina provoca cansaço físico e mental, distúrbios, privação e má qualidade do sono., 1,2

O tipo de trabalho é um dos fatores envolvidos na qualidade do sono, sendo os trabalhadores da área da saúde um dos grupos de profissionais que mais tem comprometimento da qualidade do sono. ${ }^{3}$ Uma característica do serviço prestado por esses profissionais é o trabalho em turnos, uma forma de organização do trabalho em que várias equipes se revezam para que o serviço seja ofertado continuamente, com extensão dos horários de trabalho, pela necessidade de assistência aos pacientes 24 horas por dia.4-6

Recentemente, com a instalação do quadro de pandemia de covid-19, vem sendo exigido aos profissionais de saúde o máximo de sua força de trabalho. O quadro de pandemia fez com que os serviços de saúde entrassem em situação de pressão por adequada oferta de equipamentos de proteção individual, treinamentos para uma assistência adequada, maior quantitativo de pacientes a serem atendidos e, evidentemente, maior carga de trabalho e de exposição dos profissionais de saúde a uma potencial contaminação. ${ }^{\text {? }}$

Neste contexto, os trabalhadores da saúde são submetidos a carga horária de trabalho maior e irregular, além de serem submetidos a situações de estresse e ansiedade. ${ }^{8}$ Essas situações levam a sofrimento psíquico, e tem impacto negativo na qualidade do sono desses profissionais, como mostrado em um estudo que investigou as complicações psicológicas da COVID-19. ${ }^{9}$ Assim, tornam-se importantes estudos sobre o impacto da pandemia de covid-19 e das novas rotinas de trabalho impostas ao trabalhador no estado de saúde de profissionais de saúde, em especial os fisioterapeutas hospitalares.
Com a pandemia de covid-19, houve maior necessidade de oferta de leitos de Unidade de Terapia Intensiva para a internação dos casos graves que necessitam de suporte de oxigênio e ventilação mecânica. Nesta perspectiva, os fisioterapeutas foram profissionais de linha de frente no combate à doença, devido a suas atribuições frente ao manejo de ventiladores mecânicos, gerenciando esse processo e demais recursos terapêuticos. Assim, questiona-se sobre a qualidade de sono dos profissionais de fisioterapia hospitalar que estão sob o estresse da rotina de linha de frente em combate à doença covid-19. Para responder a esse questionamento, este estudo tem como objetivo avaliar a qualidade de sono e sonolência diurna de profissionais de fisioterapia hospitalar durante o período de pandemia do covid-19.

\section{Materiais e métodos}

O estudo foi realizado de forma observacional, transversal, prospectiva, coletando dados de natureza quantitativa, sendo realizada nas dependências de um Hospital Público, localizado na cidade de Teresina-PI, Brasil. A população do estudo foi 52 profissionais de fisioterapia do referido Hospital Público. A amostra deste estudo foi constituída por todos os profissionais que se enquadraram no critério de inclusão de estar na assistência direta a pacientes internados no referido hospital entre o período de abril a setembro de 2020. Foram excluídos aqueles que se encontravam de férias ou atestado prolongado do trabalho no momento da coleta de dados e/ou não tenham preenchido por completo os instrumentos de coleta de dados. Desta forma, a amostra final foi de 45 fisioterapeutas.

Inicialmente, os pesquisadores informaram os participantes quanto aos objetivos da pesquisa e, após autorização da participação voluntária assinando o Termo de Consentimento Livre e Esclarecido, os pesquisadores enviaram instrumento de coleta de dados em formato virtual, além de ficarem disponíveis para eventuais esclarecimentos. O preenchimento se deu no horário de intervalo do trabalho ou mesmo fora das dependências do local de trabalho. A abordagem aos participantes do estudo ocorreu entre os meses de agosto e setembro de 2020. 
Foram coletadas informações dos aspectos demográficos, sociais com dados sobre idade, sexo, setor de trabalho (setores não covid e/ou covid) e se teve outros vínculos empregatícios, hábitos de saúde (prática de atividade física e consumo de estimulantes) e percepção sobre o estresse. Por sua vez, a avaliação da qualidade de sono foi feita por meio do Índice da Qualidade do Sono de Pittsburgh (IQSP), validado no Brasil. ${ }^{10}$ Este instrumento é composto por 19 itens em autorrelato e cinco itens direcionados ao acompanhante de quarto, distribuídos em sete domínios: (1) qualidade subjetiva do sono; (2) latência do sono; (3) duração do sono; (4) eficiência habitual do sono; (5) alterações do sono; (6) uso de medicações para o sono; e (7) disfunção diurna do sono. Cada item do IQSP foi medido em escala do tipo Likert de quatro pontos, cujos níveis variam de zero (nenhuma dificuldade) a três (dificuldade grave). O escore global do instrumento é obtido a partir do somatório dos escores dos sete domínios e varia de 0 (zero) a 20 pontos, de modo que maiores escores totais indicam pior qualidade do sono. O resultado pode ser classificado em: boa qualidade do sono (de 0 a 5), qualidade ruim (>5). 10

Para a avaliação da sonolência diurna excessiva, foi utilizada a Escala de Sonolência de Epworth (ESE), idealizada com base em observações relacionadas à natureza e à ocorrência da sonolência diurna, e validada no Brasil. 11 O instrumento avalia a probabilidade de adormecer em oito situações envolvendo atividades diárias, é simples, autoaplicável e de rápido preenchimento. Cada item da ESE é medido em escala do tipo Likert de quatro pontos, cujos níveis são: (0) nunca dormiria; (1) pequena chance de cochilar; (2) chance moderada de cochilar; e (3) alta chance de cochilar. O escore global é obtido por meio do somatório dos valores dos oito itens e pode variar de zero a 24 pontos. Pontuações mais elevadas correspondem a maiores graus de sonolência. Para classificação, escores acima de 10 pontos sugerem diagnóstico de sonolência diurna excessiva (SDE). ${ }^{11}$
Os dados foram organizados em planilha no programa Microsoft Excel versão 8.0 e posteriormente exportados para o programa Statistical Package for the Social Sciences (SPSS) versão 22.0, sendo as variáveis descritas por meio de porcentagem, média, mediana e desvio padrão. A análise dos dados categóricos foi feita pela medida de associação Qui-quadrado e apresentação em porcentagens (descritiva). Para análise das variáveis contínuas foi realizada a verificação da normalidade dos dados pelo teste KolmogorovSmirnov para posteriormente determinação dos testes de análise comparativa (Mann-Whitney e KruskalWallis). Foi considerado um intervalo de confiança de $95 \%$ e nível de significância de $5 \%(p<0,05)$.

Esta pesquisa ocorreu após a aprovação do Comitê de Ética em Pesquisa com CAAE: 35647020.8.0000.8050. A pesquisa esteve de acordo com as normas da resolução 466/12 do Conselho Nacional de Saúde - CNS envolvendo pesquisas em seres humanos.

\section{Resultados}

Neste estudo foram avaliados 45 fisioterapeutas e observou-se que $62,2 \%$ eram do sexo feminino, com média de idade de 39,3 $\pm 5,9$ anos (mínimo $=29$; máximo $=57$ anos), no qual $66,7 \%$ profissionais relataram carga horária semanal de trabalho de pelo menos $60 \mathrm{~h}, 55,6 \%$ trabalharam em setores covid e não covid. Sobre as atividades rotineiras que possam impactar no sono, observou-se o relato de $73,3 \%$ não praticavam atividade física regular, $44 \%$ consumiam estimulantes pelo menos 01 vez ao dia, 17,8\% fizeram uso de medicamentos para o sono, depressão ou ansiedade nos últimos 30 dias. Quanto à percepção sobre o nível de estresse nos últimos 30 dias, $48,9 \%$ relataram como moderado. A tabela 1, a seguir traz a caracterização dos fisioterapeutas atuantes no hospital durante o período de pandemia de covid-19. 
Tabela 1. Caracterização dos fisioterapeutas hospitalares no período de pandemia de covid-19, Teresina-Pl, 2020.

\begin{tabular}{|c|c|c|}
\hline Variáveis & $\mathbf{n}$ & $\%$ \\
\hline \multicolumn{3}{|l|}{ Sexo } \\
\hline Feminino & 28 & 62,2 \\
\hline Masculino & 17 & 37,8 \\
\hline \multicolumn{3}{|l|}{ Carga horária de trabalho } \\
\hline Pelo menos 30h & 15 & 33,3 \\
\hline Pelo menos $60 \mathrm{~h}$ & 30 & 66,7 \\
\hline \multicolumn{3}{|l|}{ Setores que trabalham } \\
\hline Apenas setor não covid & 10 & 22,2 \\
\hline Em setores covid e não covid & 25 & 55,6 \\
\hline Apenas em setores covid & 10 & 22,2 \\
\hline \multicolumn{3}{|l|}{ Pratica atividade física regular } \\
\hline Sim & 12 & 26,7 \\
\hline Não & 33 & 73,3 \\
\hline \multicolumn{3}{|l|}{ Consumo de estimulantes } \\
\hline Menos de 01 vez ao dia & 14 & 31,1 \\
\hline Pelo menos 01 vez ao dia & 20 & 44,4 \\
\hline 02 vezes ao dia ou mais & 11 & 24,4 \\
\hline \multicolumn{3}{|l|}{ Percepção sobre o nível de estresse } \\
\hline Nenhum & 03 & 6,7 \\
\hline Baixo & 16 & 35,6 \\
\hline Moderado & 22 & 48,9 \\
\hline Alto & 04 & 8,9 \\
\hline
\end{tabular}

$\mathrm{Na}$ análise da qualidade do sono dos profissionais de fisioterapia, observou-se um IQSP médio de 8,2 $\pm 4,0$, compatível com escore má qualidade do sono, e $68,9 \%$ de prevalência de fisioterapeutas com má qualidade do sono. Não se observou diferença entre os escores e prevalências quando analisado os grupos sexos, carga horária de trabalho, setores de trabalho e percepção do nível de estresse. A figura 1, a seguir, traz uma descrição dos escores do IQSP de forma geral, por grupo de carga horária e setor de trabalho.

Figura 1. Média dos escores do IQSP dos profissionais de fisioterapia hospitalar de forma geral, por carga horária de trabalho e setores de trabalho. Teresina-PI, 2020

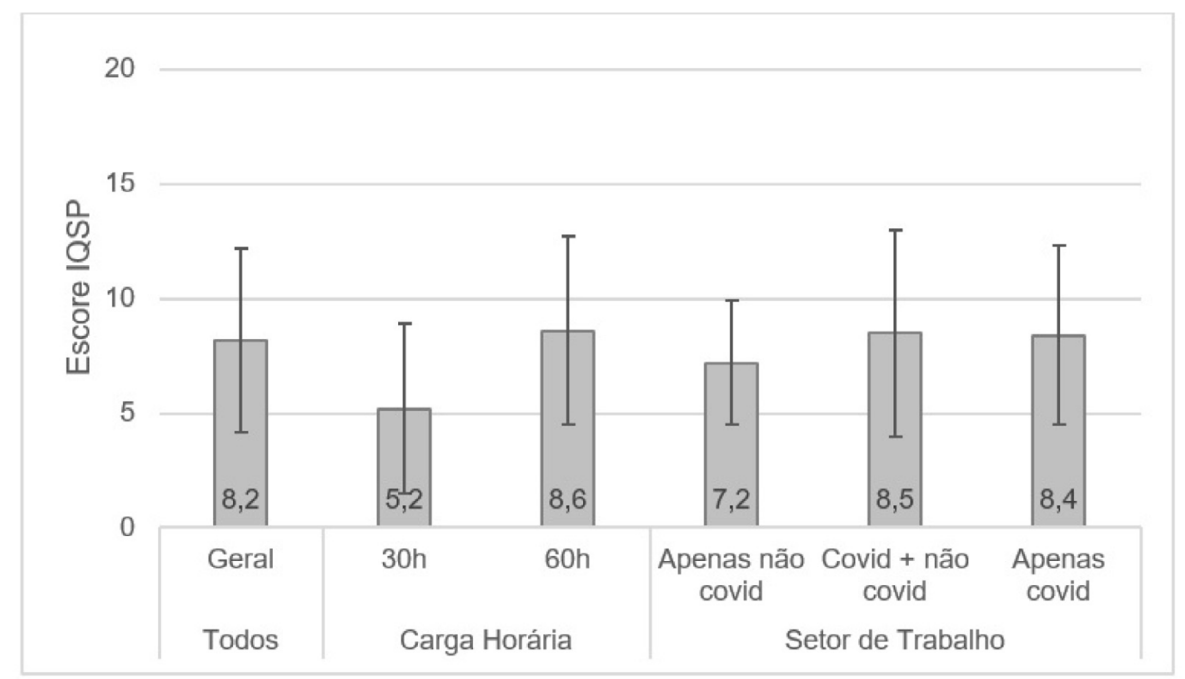

Fonte: Dados do pesquisador. 
$\mathrm{Na}$ avaliação dos achados da ESE, observou-se uma média geral de ESE de 8,3 $\pm 3,9$ (ESE > 10, presença de sonolência diurna excessiva - SDE) e prevalência de SDE de 31,1\%. Ao analisar os escores de ESE nos grupos carga horária e setores de trabalho verificou-se que na comparação entre os grupos de 30h e 60h de trabalho semanal houve diferenças nos escores com maiores valores para os profissionais que trabalhavam por maior tempo $(9,3$ $\pm 3,8$ vs $6,3 \pm 3,3 ; p=0,019$ ) como mostra a Figura 2 .

Figura 2. Média dos escores do ESE dos profissionais de fisioterapia hospitalar de forma geral, por carga horária de trabalho e setores de trabalho. Teresina-PI, 2020

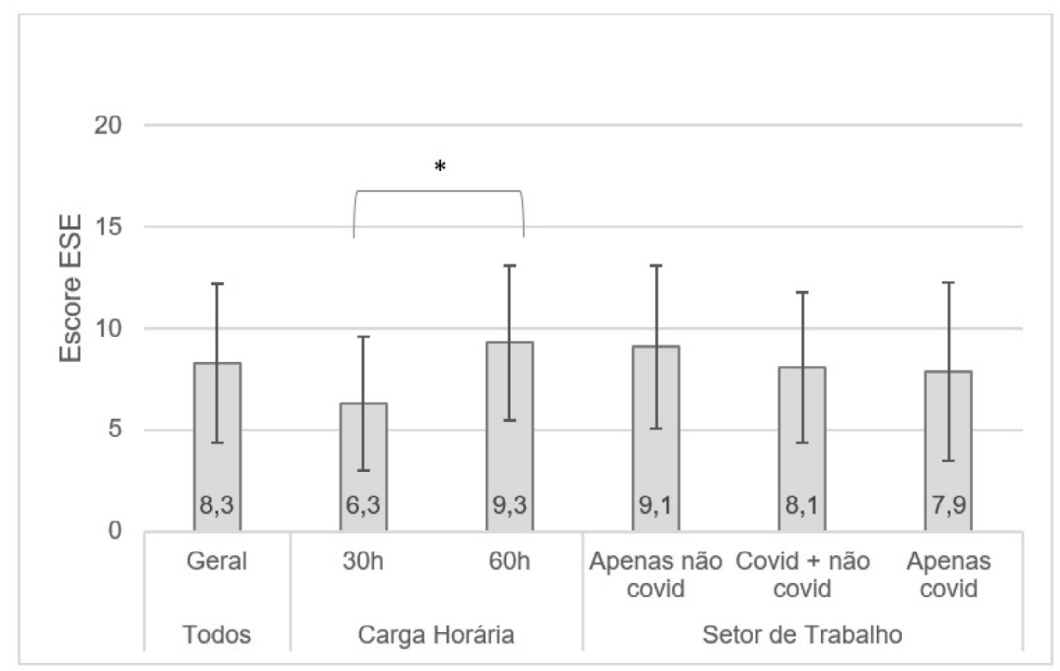

Fonte: Dados do pesquisador. Teste Mann Whitney. * $p<0,05$.

Observou-se também diferença na prevalência de SDE (X2 =6,273, $p=0,012)$ com 6,7\% vs 43,3\% para os grupos $30 \mathrm{~h}$ e $60 \mathrm{~h}$ de trabalho, respectivamente. A Figura 3 mostra as frequências de SDE para os grupos de profissionais conforme carga horária de trabalho.

Figura 3. Prevalência de profissionais com SDE conforme carga horária de trabalho. Teresina-PI, 2020

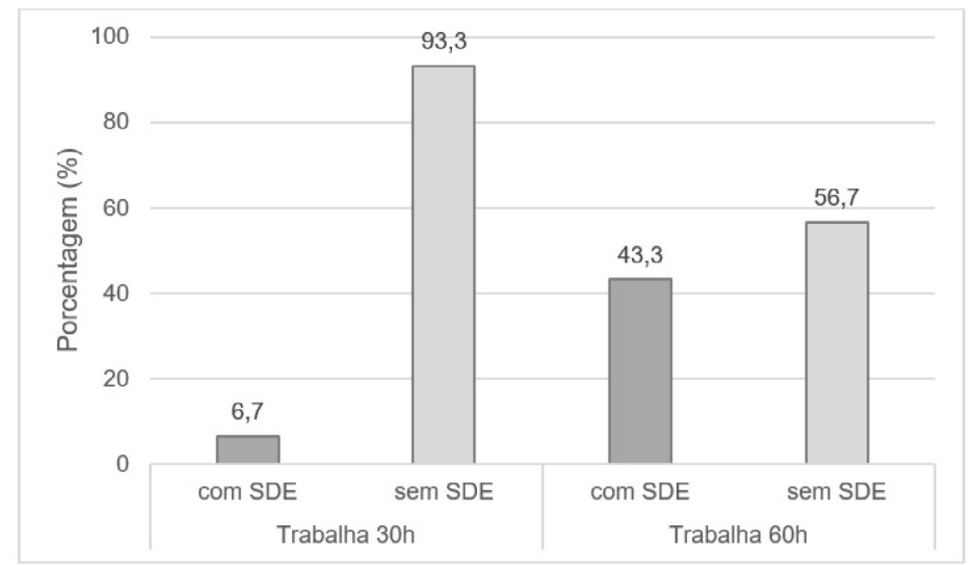

Fonte: Dados do pesquisador. 
Ainda com relação aos grupos de profissionais com base na carga horária, não se observou diferença na prevalência de percepção do estresse, uso de medicamentos e uso de estimulantes. Em outra análise comparativas entre os sexos, não houve diferença nas prevalências de má qualidade do sono, sonolência diurna excessiva e da percepção do nível de estresse. Quanto a comparação dos grupos setores de trabalho observou-se diferença apenas na percepção de estresse entre os grupos setores $(X 2=15,612 ; p=$ $0,016)$ com maior percepção de estresse moderada para o grupo do setor não covid (70\%), seguido do setor covid e não covid (52\%) e apenas setor covid (20\%). E nas demais variáveis analisadas não houve diferença (má qualidade do sono, presença SDE, uso de medicamentos para dormir, depressão/ansiedade e consumo de estimulantes).

\section{Discussão}

A presente pesquisa constatou que os fisioterapeutas atuantes em um hospital público durante a pandemia de covid-19 apresentaram, em sua maioria, carga horária de trabalho de 60 h e prestaram assistência em setores covid e não covid. Estes profissionais apresentaram elevada prevalência de má qualidade do sono. Adicionalmente, tiveram frequência importante de sonolência diurna excessiva, com maior predomínio naqueles que tiveram carga horária semanal de trabalho de $60 \mathrm{~h}$.

Com relação as características do trabalho, a maioria trabalhou cerca de $60 \mathrm{~h}$ por semana, e exercem suas atividades assistenciais tanto em locais de assistência covid e não covid. Previamente a literatura relatou que profissionais de saúde de unidades de terapia intensiva são sujeitos a uma carga de trabalho excessiva e estressante, uma vez que lidam com vidas de pacientes em risco e são cobrados a tomar medidas muitas vezes complexas. ${ }^{12}$ Além disso, trabalham em mais de um turno, em finais de semana e feriados, visto a esse contexto, a dificuldade dos profissionais da saúde, especialmente os que trabalham no período noturno, é conseguir adaptar o sono ao ritmo de vida e de trabalho colaborando para desfechos negativos à saúde. 12

Assim, a rotina hospitalar pode ser estressante para a equipe multiprofissional, justificando os resultados encontrados que evidenciaram um moderado nível de estresse nos profissionais do presente estudo.13,14 Somado a isso, nesse cenário de pandemia no qual os profissionais estão suscetíveis à contaminação durante seus atendimentos ${ }^{15}$ e com receio da possibilidade de contágio de familiares, observa-se altos índices de sofrimento psíquico, sendo eles: medo, ansiedade, depressão, angústia, sono não reparador como demonstraram estudos recentes que analisaram a saúde mental dos profissionais de saúde frente à pandemia de covid-19.14,16,17

O mesmo estudo aponta ainda que uma provável razão para o adoecimento seja a carga horária e a intensidade do trabalho dos profissionais de saúde que aumentaram pela alta demanda de casos, fazendo com que estes se submetam a mais de um vínculo empregatício com sobrecarga de trabalho, não tendo tempo suficiente para descanso adequado, estando assim, propensos a distúrbios do sono. ${ }^{14}$

Neste estudo, os fisioterapeutas apresentaram elevada prevalência de má qualidade do sono corroborando com estudo prévio com profissionais de saúde. $\frac{18}{}$ Estudos apontam que os distúrbios e a privação do sono em profissionais da saúde podem provocar fadiga, dificuldades na concentração e nas tomadas de decisões rapidamente e lidar com a morte e sofrimento agudo, acabam por desenvolver insônia primária, irregularidade do sono, insinuando que esses profissionais não têm o tempo de descanso essencial durante a semana, colocando em risco a saúde do paciente..$^{19} \mathrm{Em}$ estudo prévio com 168 enfermeiras em unidade hospitalar, observou-se má qualidade do sono e SDE dos profissionais independentemente do turno de trabalho. ${ }^{19}$ Em outro estudo com 60 enfermeiras, também observou-se má qualidade do sono e padrão de sono irregular. $\underline{20}$

Os resultados da presente pesquisa mostraram maior prevalência de SDE nos profissionais com maior carga horária de trabalho. Esse achado nos revela a relação entre o aumento da carga de trabalho e distúrbios do sono, pior qualidade do sono e SDE desses profissionais. De acordo com um estudo realizado com profissionais da saúde envolvidos no cuidado direto de pacientes com covid-19, houve uma proporção considerável de profissionais com sintomas de ansiedade, depressão, angústia, fatores que acabam por interferir na qualidade do sono e consequentemente na qualidade de vida dos trabalhadores. .0 
Outro estudo com profissionais da saúde que trabalhavam por maior tempo durante a semana mostrou que estes apresentaram uma duração do sono mais curta e por este motivo relatavam maior SDE, além disso, os profissionais também relataram maior sensação de fadiga. .11 A carga horária de trabalho excessiva com a presença de um segundo emprego entre profissionais da saúde também foi relacionada a maior SDE em um estudo realizado com enfermeiros. Durante essa jorna$\mathrm{da}$, os profissionais ficam mais tempo no ambiente de trabalho suportando as condições laborais; como consequência, há uma piora na qualidade de vida. $\frac{5}{}$ Para conciliar a carga horária de trabalho elevada, muitos profissionais trabalham no turno noturno. Profissionais de saúde que trabalham à noite relatam mais insônia e fadiga que profissionais que trabalham durante o dia. $\underline{22}$ Isso ocorre porque há uma alteração no ciclo sono-vigília deslocando o sono para o dia e o trabalho para a noite, o que interfere na regulação interna do sono. ${ }^{\underline{23}}$

Diante dos resultados encontrados, espera-se que este estudo tenha destacado a saúde dos profissionais de fisioterapia, profissional importante e de destaque durante a pandemia de covid-19 por atuar na linha de frente com a reabilitação respiratória e mobilização precoce desde a UTI até as enfermarias hospitalares. A elevada exposição desses profissionais a aerossóis contaminados devido aos aspectos inerentes da sua prática profissional e a complexidade das suas ações na assistência ventilatória dos pacientes, pode tornar essa atividade estressante, somado a isso, o envolvimento com vários vínculos de trabalho.

Este estudo teve como limitações ter sido realizado apenas em profissionais de um hospital e de uma única categoria profissional; no entanto, isso tornou as características da amostra mais homogêneas e possibilitou conhecer as implicações do trabalho sobre o sono dos fisioterapeutas.

Conclui-se que os fisioterapeutas que atuam em ambiente hospitalar de uma instituição pública apresentam elevada prevalência de má qualidade do sono, e a SDE apresentou elevada frequência nos profissionais com maior carga horária de trabalho (60h). Esses achados revelam a importância de abordagem sobre o tema, assim como orientação sobre medidas de saúde para melhorar a qualidade do sono dos profissionais fisioterapeutas. Sugere-se mais estudos sobre o tema com maior abrangência de instituições e profissionais, assim como proposta de intervenção para a melhoria da qualidade do sono.

\section{Contribuições dos autores}

Santos JS, Lima CA, Moreira WS, Monteiro PNC, Sales MC, Lima JS e Vieira CEN participaram da contribuição da confecção do projeto, coleta de dados e análise e discussão dos resultados. Ferreira LGF participou da orientação, confecção do projeto, submissão ao comitê de ética, coleta de dados e análise estatística do artigo.

\section{Conflitos de interesses}

Nenhum conflito financeiro, legal ou político envolvendo terceiros (governo, empresas e fundações privadas, etc.) foi declarado para nenhum aspecto do trabalho submetido (incluindo, mas não se limitando a subvenções e financiamentos, participação em conselho consultivo, desenho de estudo, preparação de manuscrito, análise estatística, etc.).

\section{Referências}

1. Jensen HI, Markvart J, Holst R, Thomsen TD, Larsen JW, Eg DM, et al. Shift work and quality of sleep: effect of working in designed dynamic light. Int Arch Occup Environ Health. 2016;89(1):49-61. http://dx.doi.org/10.1007/s00420-015-1051-0

2. Havekes R, Park AJ, Tudor JC, Luczak VG, Hansen RT, Ferri SL, et al. Sleep deprivation causes memory deficits by negatively impacting neuronal connectivity in hippocampal area CA1. Elife. 2016;5:e13424. http://dx.doi.org/10.7554/eLife.13424

3. Perez-Fuentes MDC, Jurado MDMM, Marquez MDMS, Martin $A B B$, Linares JJG. Emotional Effects of the Duration, Efficiency, and Subjective Quality of Sleep in Healthcare Personnel. Int. J. Environ. Res. Public Health. 2019;16(19):3512. https://dx.doi. org/10.3390\%2Fijerph16193512

4. Mendes SS, Martino MMF. Trabalho em turnos: estado geral de saúde relacionado ao sono em trabalhadores de enfermagem. Rev Esc Enferm USP. 2012;46(6):1471-6. https://doi.org/10.1590/ S0080-62342012000600026

5. Palhares VC, Corrente JE, Matsubra BB. Associação entre qualidade do sono e qualidade de vida de profissionais de enfermagem que trabalham em turnos. Rev Saúde Pública. 2014;48(4):594-601. https://doi.org/10.1590/S00348910.2014048004939

6. Rosado IVM, Russo GHA, Maia EMC. Produzir saúde suscita adoecimento? As contradições do trabalho em hospitais públicos de urgência e emergência. Ciênc saúde colet. 2015;20(10):302132. https://doi.org/10.1590/1413-812320152010.13202014

7. Travassos C. A investigação em serviços de saúde e a pandemia de covid-19. Cad. Saúde Pública. 2020;36(9):1-3. https://doi. org/10.1590/0102-311X00243920 
8. Blake H, Bermingham F, Johnson G, Tabner A. Mitigating the Psychological Impact of COVID-19 on Healthcare Workers: A Digital Learning Package. Int. J. Environ. Res. Public Health. 2020;17(9):2997. https://doi.org/10.3390/ijerph17092997.

9. Vindegaard N, Benros ME. COVID-19 pandemic and mental health consequences: systematic review of the current evidence. Brain Behav Immun. 2020;89:531-42. https://doi.org/10.1016/j. bbi.2020.05.048

10. Bertolazi AN, Fagondes SC, Hoff LS, Dartora EG, Miozzo ICS, Barba MEF, et al. Validation of the Brazilian Portuguese version of the Pittsburgh Sleep Quality Index. Sleep Med. 2011;12(1):70-5. https://doi.org/10.1016/j.sleep.2010.04.020

11. Bertolazi AN, Fagondes SC, Hoff LS, Pedro VD, Barreto SSM, Johns MW. Validação da escala de sonolência de Epworth em português para uso no Brasil. J Bras Pneumol. 2009;35(9):877-83. https://doi.org/10.1590/S1806-37132009000900009

12. Carvalhais FR, Moreira AM, Mendonça RL, Ottano C. Frequência da síndrome de Burnout em uma Unidade de Terapia Intensiva: uma perspectiva multiprofissional. Rev. Pre. Infec e Saúde. 2015;1(4):1-10. https://doi.org/10.26694/repis. v1i 4.4271

13. Silva JSX, Silva RM, Cangussu DDD, Moraes Filho IM, Pérez MA, Proença MFR. Qualidade do sono dos profissionais de enfermagem do serviço móvel de atendimento de urgência. REVISA. 2019;8(3):264-72. https://doi.org/10.36239/revisa.v8.n3. p264a272

14. Cabral JVB, Neves SC, Oliveira FHPC. Estresse dos profissionais de enfermagem em Unidade de Terapia Intensiva (UTI). Rev eletrônica Diálogos Acadêmicos [Internet]. 2016;11(2):3342. Disponível em: http://uniesp.edu.br/sites/_biblioteca/ revistas/20170627114039.pdf

15. Rowlands AV, Gillies C, Chudasama Y, Davies MJ, Islam N, Kloecker DE, et al. Association of working shifts, inside and outside of healthcare, with severe COVID-19: an observational study. BMC Public Health. 2021;21:773. https://doi.org/10.1186/ s12889-021-10839-0
16. Lai J, Ma S, Wang Y, Cai Z, Hu J, Wei N, et al. Factors Associated With Mental Health Outcomes Among Health Care Workers Exposed to Coronavirus Disease 2019. JAMA Netw Open. 2020;3(3):e203976. https://doi.org/10.1001/ jamanetworkopen.2020.3976

17. Prado AD, Peixoto $B C$, Silva AMB, Scalia LAM. A saúde mental dos profissionais de saúde frente à pandemia do COVID-19: uma revisão integrativa. Rev. Eletrônica Acervo Saúde. 2020;(46):e4128. https://doi.org/10.25248/reas.e4128.2020

18. Martin AHS, Serrano JP, Cambriles TD, Arias EMA, Mendez JM, Alvarez MJY, et al. Sleep characteristics in health workers exposed to the COVID-19 pandemic. Sleep Med. 2020;75:388-94. https:// doi.org/10.1016/j.sleep.2020.08.013

19. Guerra PC, Oliveira NF, Sande MT, Terreri LRA, Len CA. Sono, qualidade de vida e humor em profissionais de enfermagem em Unidades de Terapia Intensiva Infantil. Rev Esc Enferm USP. 2016;50(2):279-85. http://dx.doi.org/10.1590/S0080623420160000200015

20. Martino MMF, Abreu ACB, Barbosa MFS, Teixeira JEM. Relação entre trabalho por turnos e padrões de sono em enfermeiros. Ciênc Saúde Coletiva. 2013;18(3):763-8. https://doi.org/10.1590/ S1413-81232013000300022

21. Teixeira CFS, Soares CM, Souza EA, Lisboa ES, Pinto ICM, Andrade LR, et al. A saúde dos profissionais de saúde no enfrentamento da pandemia de Covid-19. Ciênc Saúde Coletiva. 2020;25(9):3465-74. https://doi.org/10.1590/1413$\underline{81232020259.19562020}$

22. Chaiard J, Deeluea J, Suksatit B, Songkham W, Inta N. Short sleep duration among Thai nurses: Influences on fatigue, daytime sleepiness, and occupational errors. J. Occup. Health. 2018;60(5):348-55. https://dx.doi.org/10.1539\%2Fjoh.2017-0258$\mathrm{OA}$

23. Oyane NMF, Pallesen S, Moen BE, Akerstedt T, Bjorvatn B. Associations between night work and anxiety, depression, insomnia, sleepiness and fatigue in a sample of Norwegian nurses. PloS one. 2013;8(8):e70228. https://doi.org/10.1371/ journal.pone.0070228 